\title{
Melanonychia in Patients Infected with Human Immunodeficiency Virus Original Communication
}

\author{
Parvaneh Ehsanzadeh-Cheemeh ${ }^{1}$, Richard M. Grimes ${ }^{2}$, Paul Rowan ${ }^{3}$, Yu-Jing Huang ${ }^{3}$, E. James Essien ${ }^{1}$, \\ Stanley T. Lewis ${ }^{4}$ \\ ${ }^{1}$ The Institute of Community Health, College of Pharmacy, University of Houston, Houston, USA; ${ }^{2}$ The University of Texas Health \\ Science Center at Houston Medical School, Department of Internal Medicine, Houston, USA; ${ }^{3}$ The University of Texas Health Sci- \\ ence Center at Houston, School of Public Health, Houston, USA; ${ }^{4}$ TaiMed Biologics, Inc., Houston, USA. \\ Email: richard.m.grimes@uth.tmc.edu
}

Received September 17 $7^{\text {th }}, 2011$; revised October 19 ${ }^{\text {th }}, 2011$; accepted October $31^{\text {st }}, 2011$.

\begin{abstract}
Study Purposes. This study examined whether melanonychia was more prevalent in 1) HIV positive individuals compared to HIV negative persons, 2) HIV positives exposed to zidovudine and/or stavudine and 3) those with darker skin pigmentation. Procedures. 267 HIV positive and 273 HIV negative patients were examined for presence or absence of melanonychia and level of skin pigmentation using the Fitzgerald scale. Pharmacy records were examined for determining exposure to zidovudine or stavudine. Chi square, odds ratios and logistic regression were used to examine the study questions Main Findings. Melanonychia appeared in 49.1\% of 267 HIV positive and 21.8\% of 273 HIV negative subjects. Adjusting for skin pigmentation, HIV positives were 4.1 times more likely to have melanonychia than HIV negatives. Melanonychia was present in 54\% of those receiving zidovudine and in $42 \%$ of those receiving stavudine $(O R=2.73, p=0.05)$. In a multivariate model in HIV positives which included skin type, prescription of zidovudine and/or Stavudine, only dark skin $(O R=14.62, p<0.001)$ and zidovudine $(O R=2.65, p<0.03)$ were significant. Principal Conclusions. HIV infected persons are prone to melanonychia. This is more frequent in darker skinned persons and is enhanced in those exposed to zidovudine.
\end{abstract}

Keywords: Zidovudine, Stavudine, Melanonychia, HIV/AIDS, Skin Pigmentation

\section{Introduction}

Antiretroviral therapy (ART) changed HIV infection from being a death sentence to a manageable chronic disease. However, in order for the drugs to maintain their effecttiveness, patients must rigorously adhere to their drug regimens. However, patients often fail to adhere to these medications. Non-adherence has been attributed to a number of reasons including patients' not believing in the efficacy of ART, lack of social support, irregular clinic visits, drug abuse, alcohol addiction, psychiatric disease (especially depression), young age, low literacy and medication side effects [1]. Therefore, potential events that may cause adherence problems need to be anticipated and dealt with prior to their occurrence. One problem that has been shown to cause poor adherence in a variety of settings is concern over the negative changes in physical appearances that are perceived to be associated with ART. This has been noted in the United States [2-5], Poland [6], Brazil [7], Italy [8], and Germany [9]. HIV infected persons think that appearance changes stigmatize them [10].

A readily visible and frequently occurring change in appearance of HIV infected persons is the darkening of the fingernails and toenails [11-14]. This is called melanonychia, a condition that is characterized by longitudenal hyper pigmented lines in the nails. Pictures of this condition can be found on the internet [15]. The increase in nail pigmentation is caused by amplified pigment production by melanocytes, which is due to activation of nail matrix melanocytes. The mechanism of nail melanocytes activation is not fully known but it is believed to be partly due to over expression of $\alpha$-melanocyte-stimulating hormone and adrenocorticotropic hormone activity as well as ultraviolet light. Melanonychia usually reflects the presence of a benign lesion within the matrix caused by a melanocytic nevus, simple lentigo, or increased activation of benign melanocytes [16-18]. However, it can also represent a number of different pathologic conditions including bacterial or fungal infections, melanoma and other cancers [19]. It is also associated with certain 
medical procedures including chemotherapy and the use of certain medications [20,21].

Melanonychia is reported to be more prevalent in dark-skinned individuals such as persons of African origin, Hispanics, and Asians [21]. Also, melanonychia has been associated with the use of zidovudine, a commonly used anti-HIV drug which is a thymidine analog from the nucleoside reverse transcriptase inhibitor class of drugs [22-25]. There is one other commonly used thymidine analog, nucleoside reverse transcriptase inhibitor which is called stavudine. No studies were found that investigated whether stavudine was associated with melanonychia. So, it is not clear whether the presence of melanonychia in HIV infected person is due to skin tone, HIV infection itself, or to taking zidovudine and/or stavudine or the interaction of any or all of these factors. Therefore, a study was conducted to evaluate the prevalence of melanonychia in: 1) HIV infected individuals as compared to infected individuals who were not HIV-infected; 2) HIV infected individuals who were exposed to zidovudine and/or stavudine compared to those who were not, and 3) persons with various levels of skin pigmentation.

\section{Methods}

After approval was obtained from the Committee for the Protection of Humans Subjects of The University of Texas Health Science Center, (the institutional review board), 267 HIV positive patients were examined to collect information on presence or absence of melanonychia, skin pigmentation, and exposure to zidovudine or stavudine. Information on presence or absence of melanonychia and skin pigmentation was collected from 273 HIV-negative volunteers of similar age and gender who were used for comparison in the study. The HIV negative subjects were being seen for other medical conditions at the same center.

Both groups were examined using a standardized examination protocol, whereby the attending clinician assessed the presence or absence of melanonychia by the examining all twenty nail beds. Skin type was determined by physical inspection and was classified using the Fitzpatrick Classification Scale [26]. The scale ranks the darkness of skin on a one to six scale with six being the score assigned to those with the most pigmented skin. In order to have a sufficient sample size in each of the skin type groups, the patients were combined into three groups for analysis purposes. Those with a Fitzgerald score of 1 and 2 were analyzed as one group, those with scores of 3 and 4 in a second group and those with score of 5 and 6 in the third group.

Demographics, HIV status and skin type were also examined to determine the relative frequency of each in patients with and without melanonychia. A logistic regression was used to determine likelihood of melanonychia being dependent upon the predictors of interest: HIV status, skin coloration type, and whether the patient was exposed to stavudine or zidovudine. Because some of the patients had received both of the drugs during different times, an interaction term for patients receiving both of these medications was also included to see if there was an additive effect. Another logistic regression model was developed to determine the likelihood of melanonychia associated with skin color for HIV positive participants only.

\section{Results}

Table 1 reports the demographic characteristics and skin types of the group with HIV infection and the group that was HIV free. The two groups were remarkably similar with regard to race, gender and skin tones. Table 2 illustrates the demographic characteristics of patients with and without melanonychia. There were no statistically significant differences with regard to gender or age. There were differences across race/ethnicity. Melanonychia was found in 156 of 275 (56.7\%) of African-Americans and in 30 of $155(19.4 \%)$ Hispanics. In contrast it was found in only 5 of 110 (4.5\%) Caucasians. Persons with skin types 5 and 6 were far more likely to have melanonychia (104 out of 242 or $57.9 \%$ ) than those with skin types 3 and 4 (44 out of 206 or $21.4 \%)$. Only five out of $92(5.4 \%)$ of those with skin types 1 and 2 had melanonychia. Persons with HIV infection were more likely to have melanonychia (131 out of 267 or $49.1 \%$ ) than those who were not infected with HIV (60 out of 273 or $21.8 \%$ ).

Table 3 presents the number and percent of these patients with HIV infection who were prescribed stavudine, zidovudine, both drugs and neither of the drugs. Melanonychia was found in 39 out of 72 (54\%) of patients who had exposure to zidovudine while it occurred in 24 of 58

Table 1. Demographic and skin type characteristics of HIV positive and HIV negative subjects.

\begin{tabular}{lccc}
\hline \multicolumn{1}{c}{ Total =540 } & $\begin{array}{c}\text { HIV Positive } \\
\text { N }=267\end{array}$ & $\begin{array}{c}\text { HIV Negative } \\
\text { N = 273 }\end{array}$ & $P$ value \\
\hline Age (Mean) & $40 y r s$ & $40 y r s$ & 0.92 \\
Race & 51 & 59 & \\
White & 143 & 132 & 0.50 \\
African-American & 73 & 83 & 0.50 \\
Hispanic & & & 0.30 \\
Skin Type(FCS) & 42 & 50 & 0.40 \\
1 \& 2 & 99 & 107 & 0.60 \\
3 \& 4 & 126 & 116 & 0.52 \\
5 \& 6 & & & \\
Gender & $202(75.7 \%)$ & $204(74.7 \%)$ & 0.80 \\
Male & $65(24.3 \%)$ & $69(25.3 \%)$ & 0.90 \\
Female & & & \\
\hline
\end{tabular}

*Fitzgerald classification scale. 
Table 2. Demographics and clinical characteristics of subjects with and without melanonychia, number (\%) or mean (standard).

\begin{tabular}{|c|c|c|c|c|c|}
\hline \multirow{2}{*}{ Total $=540$} & \multicolumn{2}{|c|}{$\begin{array}{l}\text { No Melanonychia } \\
\qquad(\mathrm{N}=349)\end{array}$} & \multicolumn{2}{|c|}{$\begin{array}{l}\text { Melanonychia } \\
(\mathrm{N}=191)\end{array}$} & \multirow[t]{2}{*}{$P$ value } \\
\hline & (n) & $(\%)$ & (n) & (\%) & \\
\hline \multicolumn{6}{|l|}{ Age Groups } \\
\hline$<39$ & 177 & 50.72 & 79 & 41.40 & $<0.01$ \\
\hline $40-49$ & 113 & 32.40 & 70 & 36.70 & $<0.01$ \\
\hline $50-59$ & 44 & 12.61 & 31 & 16.23 & 0.13 \\
\hline$>=60$ & 15 & 4.30 & 11 & 5.80 & 0.43 \\
\hline Test Across Age Groups & & & & & 0.20 \\
\hline \multicolumn{6}{|l|}{ Race } \\
\hline White & 105 & 30.10 & 5 & 2.62 & $<0.01$ \\
\hline African-American & 119 & 34.10 & 156 & 81.70 & $<0.03$ \\
\hline Hispanic & 125 & 35.82 & 30 & 15.71 & $<0.01$ \\
\hline Test Across Race & & & & & $<0.01$ \\
\hline \multicolumn{6}{|l|}{ Gender } \\
\hline Females & 91 & 26.10 & 43 & 22.51 & $<0.01$ \\
\hline Males & 258 & 73.93 & 148 & 77.50 & $<0.01$ \\
\hline Test Across Gender & & & & & 0.36 \\
\hline \multicolumn{6}{|l|}{ HIV } \\
\hline Negative & 213 & 61.03 & 60 & 31.41 & $<0.01$ \\
\hline Positive & 136 & 39.00 & 131 & 68.60 & 0.76 \\
\hline Test Across HIV Status & & & & & $<0.01$ \\
\hline \multicolumn{6}{|l|}{ Skin Type } \\
\hline $1 \& 2$ & 85 & 24.40 & 7 & 3.70 & $<0.01$ \\
\hline $3 \& 4$ & 162 & 46.42 & 44 & 23.04 & $<0.01$ \\
\hline $5 \& 6$ & 102 & 29.23 & 140 & 73.30 & $<0.01$ \\
\hline Test Across Skin Type & & & & & $<0.01$ \\
\hline
\end{tabular}

Table 3. Presentation of melanonychia and NRTIs prescription use by HIV positive patients.

\begin{tabular}{lcccc}
\hline \multicolumn{1}{c}{ Total $=267$} & \multicolumn{2}{c}{$\begin{array}{c}\text { No Melanonychia } \\
\mathrm{N}=136\end{array}$} & \multicolumn{2}{c}{$\begin{array}{c}\text { Melanonychia } \\
\mathrm{N}=131\end{array}$} \\
\hline & $\mathrm{(n)}$ & $(\%)$ & $(\mathrm{n})$ & $(\%)$ \\
Zidovudine & 33 & 24.30 & 39 & 29.80 \\
Stavudine & 34 & 25.00 & 24 & 18.23 \\
Zidovudine and Stavudine & 48 & 35.30 & 52 & 39.70 \\
None & 21 & 15.44 & 16 & 12.21 \\
\hline
\end{tabular}

(42\%) patients who had taken stavudine. Of those who had taken both zidovudine and stavudine, 52 out of $100(52 \%)$ patients were diagnosed with melanonychia.

Table 4 presents the results of the logistic regression model which adjusted for skin type. Participants with melanonychia were 4.1 times more likely to be from the group with HIV infection compared to the group without HIV infection (OR $=4.1 ; 95 \%$ CI, $2.7-6.35 ; p<0.001)$. Melanonychia was more likely to be found among participants with darker skin coloration. Participants with melanonychia were more likely to be in the medium coloration group compared to the lighter skin coloration group (OR $=3.4 ; 95 \% \mathrm{CI}, 1.4-8.0 ; p<0.001)$, and participants with melanonychia were far more likely to be in the darker skin coloration group compared to the medium skin coloration group $(\mathrm{OR}=19.5 ; 95 \% \mathrm{CI}, 8.4-45.0 ; p$ $<0.001)$.

Table 5 illustrates the outcomes of the second logistic regression model, with likelihood of melanonychia as predicted by skin coloration, and by prescription or nonprescription of either medication, and the interaction of prescription of both. Patients with HIV infection who were diagnosed with melanonychia were at 2.6 greater odds of being from skin group 3 - 4 than from skin types 1 - 2. Patients with HIV infection who were diagnosed with melanonychia were at 14.6 greater odds of being from skin types 4 - 5 than from skin type groups $1-2$. HIV Patients who had taken zidovudine were 2.6 times more likely to have melanonychia. If they had been exposed to both zidovudine and stavudine there was no difference in the presence of melanonychia. Unfortunately, the length of time of zidovudine exposure was not available. However, it seemed likely that patients who had received both drugs had been started on zidovudine and were switched to stavudine due to zidovudine intolerance and the exposure would have been brief.

Table 4. Relationship of presence of melanonychia and HIV status or skin type.

\begin{tabular}{cccc}
\hline Exposure & Adjusted Odds Ratio & $95 \% \mathrm{CI}$ & $P$ value \\
\hline HIV $(\mathrm{N}=540)$ & & & \\
Negative & 1.0 (Reference) & 1.0 (Reference) & \\
Positive & 4.14 & $2.71-6.35$ & $<0.001$ \\
Skin type $(\mathrm{N}=540)$ & & & \\
$1 \& 2$ & 1.0 (Reference) & 1.0 (Reference) & \\
$3 \& 4$ & 3.40 & $1.44-8.00$ & $<0.001$ \\
$5 \& 6$ & 19.50 & $8.44-45.00$ & $<0.001$ \\
\hline
\end{tabular}

Table 5. Melanonychia in HIV positive patients, as predicated by skin type and nucleoside reverse transcriptase inhibitor exposure $(\mathrm{N}=267)$.

\begin{tabular}{cccc}
\hline Exposure & $\begin{array}{c}\text { Adjusted Odds } \\
\text { Ratio }\end{array}$ & $95 \%$ CI & $P$ value \\
\hline Skin type & 1.0 (Reference) & 1.0 (Reference) & \\
$1 \& 2$ & 2.63 & $1.05-6.62$ & 0.04 \\
$3 \& 4$ & 14.62 & $5.70-36.00$ & $<0.001$ \\
$5 \& 6$ & & & \\
Zidovudine use & 1.0 (Reference) & 1.0 (Reference) & 0.03 \\
No & 2.65 & $1.07-6.55$ & \\
Yes & & & \\
Stavudine use & 1.0 (Reference) & 1.0 (Reference) & 0.75 \\
No & 1.16 & $0.46-2.96$ & \\
Yes & & & \\
No & 1.0 (Reference) & 1.0 (Reference) & 0.62 \\
Yes & 0.74 & $0.23-2.40$ & \\
\hline
\end{tabular}




\section{Discussion}

This study demonstrates that melanonychia is highly prevalent in HIV infected individuals with pigmented skin. Given the much higher rate in HIV infected persons than in the uninfected, it is quite possible that it will occur after infection. As a result patients may connect it to their HIV infection or to their taking antiretroviral medications. Patients may be concerned that it is a serious medical condition and/or they may think that it is cosmetically displeasing. As a result patients may question the clinician as to its origin and cause. Reassurance as to its benign nature may help the patient to cope with their medical concerns. However, patients may discover that melanonychia has been associated with zidovudine use on the internet and/or through information provided by nongovernmental organizations. If patients believe that zidovudine or other HIV medications are causing a disfigurement of his or her nails, they may stop taking that medication with negative consequences to their overall well being and a reduction of their future treatment options. Physicians should be alert to this possibility in patients who raise questions about their melanonychia and should reassure patients that it has not been linked to any medication except zidovudine. If the patient is taking zidovudine there are many other medications that can be substituted for zidovudine (including stavudine).

Because patients may link melanonychia to antiretroviral therapy (even if they are not taking zidovudine) clinicians may wish to forewarn their patients that nail changes might may occur and that it is not a reason to stop taking their antiretrovirals. In forewarning or reassuring them, clinicians should emphasize that the condition is common in those who not HIV infected (21.8\% of those who were not infected in our study) and so its appearance should not be seen as something that will reveal their HIV status nor is it a reasons to stop taking their antiretrovirals. If a patient who is taking zidovudine has the condition and is concerned about it and insists on changing medications they should be made aware that it will take a long time for the lesions to disappear.

Clinicians should also recognize that while melanonychia is almost always a benign condition that is idiopathic in origin, it is occasionally linked to more serious conditions, particularly melanoma. Tosti et al. [20] offered several clinical clues as to whether melanonychia requires additional investigation for the presence of melanoma. These were 1) discoloration bands in the nails that are wider than $3 \mathrm{~mm}$ and have irregular borders; 2) extension of the band into the proximal and/or lateral nail fold (Hutchinson's sign); 3) a lesion with a triangular shape; 4) nails that are split or have fissures; and 5) if the pigmentation is not homogenous.
Given the extremely high prevalence of melanonychia in these HIV positive patients $(>49 \%)$ clinicians might consider its presence as a potential clue to HIV positivity in previously untested patients. Alone or together with other signs or patient history, it might induce a discussion of the need to rule out HIV infection. It is seldom that a clinician has such a visible sign that might be indicative of undiagnosed HIV infection.

\section{Acknowledgements}

This study was supported by the Baylor-UT Houston Center for AIDS Research (CFAR), a program funded by the US National Institutes of Health (NIH) (AI036211).

\section{REFERENCES}

[1] A. K. Patel and K. K. Patel, "Future Implications: Compliance and Failure with Antiretroviral Treatment," Journal of Post-Graduate Medicine, Vol. 52, No. 3, 2006, pp. 197-200.

[2] T. Hawkinsm, "Appearance-Related Side Effects of HIV-1 Treatment," Aids Patient Care and STDs, Vol. 20, No. 1, 2006, pp. 6-18. doi:10.1089/apc.2006.20.6

[3] R. Power, H. L. Tate, S. M. McGill and C. Taylor, "A Qualitative Study of the Psychosocial Implications of Lipodystrophy Syndrome on HIV Positive Individuals," Sexually Transmitted Infections, Vol. 4, No. 2, 2003, pp. 137-141. doi:10.1136/sti.79.2.137

[4] I. B. Corless, K. M. Kirksey, J. Kemppainen, et al., "Lipodystrophy-Associated Symptoms and Medication Adherence in HIV-AIDS," AIDS Patient Care and STDs, Vol. 19, No. 9, 2005, pp. 577-586. doi:10.1089/apc.2005.19.577

[5] N. R. Reynolds, J. L. Neidig, A. W. Wu, A. L. Gifford and W. C. Holmes, "Balancing Disfigurement and Fear of Disease Progression: Patient Perceptions of HIV Body Fat Redistribution," AIDS Care, Vol. 18, No. 7, 2006, pp. 663-673. doi:10.1080/09540120500287051

[6] D. Rogowska-Szadkowska, S. Chlabicz, M. A. Oltarzewska and J. Sawicka-Powierza, "Which Factors Hinder the Decision of Polish HIV-Positive Patients to Take Up Antiretroviral Therapy?" AIDS Care, Vol. 21, No. 3, 2009, pp. 280-283. doi:10.1080/09540120802241871

[7] C. P. Santos, Y. X. Felipe, P. E. Braga, D. Ramos, R. O. Lima and A. C. Segurado, "Self-Perception of Body Changes in Persons Living with HIV/AIDS: Prevalence and Associated Factors," AIDS, Vol. 19, 2005, pp. S14-21. doi:10.1097/01.aids.0000191485.92285.c7

[8] A. Ammassari, A. Antinor, A. Cozzi-Lepri, et al., "Relationship between HAART Adherence and Adipose Tissue Alterations," Journal of Acquired Immune Deficiency Syndromes and Human Retrovirology, Vol. 31, 2002, pp. S140-144.

[9] M. Oette, P. Juretzko, A. Kroidl, et al., "Lipodystrophy Syndrome and Self-Assessment of Well-Being and Physical Appearance in HIV-Positive Patients," AIDS Patient 
Care and STDs, Vol. 16, No. 9, 2002, pp. 413-417. doi:10.1089/108729102760330254

[10] B. E. Berger, C. E. Ferrans and F. R. Lashley, "Measuring Stigma in People with HIV: Psychometric Assessment of the HIV Stigma Scale," Research in Nursing \& Health, Vol. 24, No. 6, 2001, p. 518. doi:10.1002/nur.10011

[11] B. Cribier, M. Mena, D. Rey, M. Partisani, V. Fabien, J. Lang, et al., "Nail Changes in Patients Infected with $\mathrm{Hu}-$ man Immunodeficiency Virus: A Prospective Controlled Study," Archive of Dermatology, Vol. 134, No. 10, 1998, pp. 1216-1220. doi:10.1001/archderm.134.10.1216

[12] B. Fisher and L. Warner, "Cutaneous Manifestations of the Acquired Immunodeficiency Syndrome," International Journal of Dermatology, Vol. 26, No. 6, 1987, pp. 615630. doi:10.1111/j.1365-4362.1987.tb02267.x

[13] A. P. Panwalker, "Nail Pigmentation in the Acquired Immunodeficiency Syndrome," Annals of Internal Medicine, Vol. 107, No. 6, 1987, pp. 943-944.

[14] P. Chandrasekar, "Nail Discoloration and Human Immunodeficiency Virus Infection," American Journal of Medicine, Vol. 86, No. 4, 1989, pp. 506-507. doi:10.1016/0002-9343(89)90365-3

[15] PicSearch. Pictures of Longitudinal Melanonychia, 2011. http://www.picsearch.com/pictures/Health/Cutaneous\%20 conditions/Longitudinal\%20melanonychia.html

[16] K. J. Smith, H. G. Skelton, J. Yeager, R. Ledsky, W. McCarthy, D. Baxter, et al., "Cutaneous Findings in HIV-1-Positive Patients: A 42-Month Prospective Study, Military Medical Consortium for the Advancement of Retroviral Research," Journal of the American Academy of Dermatology, Vol. 31, No. 5, 1994, pp. 746-754. doi:10.1016/S0190-9622(94)70236-5

[17] D. A. Glaser and K. Remlinger, "Blue Nails and Acquired Immunodeficiency Syndrome: Not Always Associated with Azidothymidine Use," Cutis, Vol. 57, No. 4, 1996, pp. 243-244.
[18] B. M. Piraccini, M. Iorizzo, A. Antonucci and A. Tosti, "Drug-I Induced Nail Abnormalities," Expert Opinion on Drug Safety, No. 1, 2004, pp. 57-65. doi: $10.1517 / 14740338.3 .1 .57$

[19] E. Haneke and R. Baran, "Longitudinal Melanonychia," Dermatologic Surgery, Vol. 27, No. 6, 2001, pp. 580-584. doi:10.1111/j.1524-4725.2001.01916.x

[20] R. Baran and P. Kechijian, "Longitudinal Melanonychia (Melanonychia Striata): Diagnosis and Management," Journal of the American Academy of Dermatology, Vol. 21, No. 6, 1989, pp. 1165-1175. doi:10.1016/S0190-9622(89)70324-8

[21] A. Tosti, Piraccini, M. Bianca and D. C. De Farias, "Dealing with Melanonychia, Seminars in Cutaneous," Medicine and Surgery, Vol. 28, No. 1, 2009, pp. 49-54.

[22] A. Tosti, G. Gaddoni, P. A. Fanti, A. D'Antuono and F. Albertini, "Longitudinal Melanonychia Induced by 3'Azidodeoxythymidine: Report of 9 Cases," Dermatologica, Vol. 180, No. 4, 1990, pp. 217-220. doi: $10.1159 / 000248033$

[23] R. G. Greenberg and T. G. Berger, "Nail and Mucocutaneous Hyperpigmentation with Azidothymidine Therapy," Journal of the American Academy of Dermatology, Vol. 22, No. 2, 1990, pp. 327-330. doi:10.1016/0190-9622(90)70039-K

[24] G. Rahav and S. Maayan, "Nail Pigmentation Associated with Zidovudine: A Review and Report of a Case," Scandinavian Journal of Infectious Diseases, Vol. 24: No. 5, 1992, pp. 557-561. doi:10.3109/00365549209054640

[25] J. Sahai, B. Conway, D. Cameron, G. Garber, "Zidovudine-Associated Hypertrichosis and Nail Pigmentation in an HIV-Infected Patient," AIDS, Vol. 5, No. 1, 1991, pp. 1395-1396. doi:10.1097/00002030-199111000-00025

[26] H. Brannon, Fitzpatrick Classification Scale, 2008. http://dermatology.about.com/od/cosmeticprocedure/a/fitz patrick.htm. 